\title{
Wakefulness Affects Synaptic and Network Activity by Increasing Extracellular Astrocyte-Derived Adenosine
}

\author{
L. Ian Schmitt, ${ }^{1}$ Robert E. Sims,${ }^{2}$ Nicholas Dale, ${ }^{2}$ and Philip G. Haydon ${ }^{1}$ \\ ${ }^{1}$ Department of Neuroscience, Tufts University Medical School, Boston, Massachusetts 02111 and ${ }^{2}$ School of Life Sciences, University of Warwick, \\ Coventry CV4 7AL, United Kingdom
}

Loss of sleep causes an increase in sleep drive and deficits in hippocampal-dependent memory. Both of these responses are thought to require activation of adenosine A1 receptors (adorA1Rs) and release of transmitter molecules including ATP, which is rapidly converted to adenosine in the extracellular space, from astrocytes in a process termed gliotransmission. Although it is increasingly clear that astrocyte-derived adenosine plays an important role in driving the homeostatic sleep response and the effects of sleep loss on memory (Halassa et al., 2009; Florian et al., 2011), previous studies have not determined whether the concentration of this signaling molecule increases in response to wakefulness. Here, we show that the level of adorA1R activation increases in response to wakefulness in mice (Mus musculus). We found that this increase affected synaptic transmission in the hippocampus and modulated network activity in the cortex. Direct biosensor-based measurement of adenosine showed that the net extracellular concentration of this transmitter increased in response to normal wakefulness and sleep deprivation. Genetic inhibition of gliotransmission prevented this increase and attenuated the wakefulness-dependent changes in synaptic and network regulation by adorA1R. Consequently, we conclude that wakefulness increases the level of extracellular adenosine in the hippocampus and that this increase requires the release of transmitters from astroctyes.

\section{Introduction}

Insufficient sleep negatively impacts cognitive function and has been shown to disrupt hippocampal-dependent memory in both rodents and humans (Graves et al., 2003; Yoo et al., 2007). Sleep need is homeostatically regulated with increased periods of wakefulness producing heightened drive to sleep as well as increased markers of sleep pressure both globally (Basheer et al., 2004) and locally (Vyazovskiy et al., 2011). The homeostatic sleep response is thought to depend upon activation of neuronal adenosine A1 (adorA1) and A2A (adorA2A) receptors. The importance of extracellular adenosine signaling to the sleep homeostat is evident in the observation that the increase in sleep pressure normally occurring in response to sleep deprivation is significantly attenuated in conditional knock-out or knock-down mice with reduced adorA1 in the CNS (Thakkar et al., 2003; Bjorness et al., 2009) and by pharmacological inhibition of this receptor (Van Dort et al., 2009). We have previously shown that mice show deficits in sleep homeostasis similar to those produced by inhibiting adorA1Rs when SNARE function is disrupted specifically in astrocytes through dominant-negative expression of the cyto-

\footnotetext{
Received Nov. 11, 2011; revised Jan. 20, 2012; accepted Feb. 10, 2012.

Author contributions: L.I.S. and P.G.H. designed research; L.I.S. performed research; R.E.S. and N.D. contributed unpublished reagents/analytic tools; L.I.S. analyzed data; L.I.S., R.E.S., N.D., and P.G.H. wrote the paper.

This work was supported by grants from the National Institutes of Health (NIH) including NIH Grants NS037585, NS054770, DA025967, and F31MH090706 - 01A1, and by the Welcome Trust UK.

P.G.H. has equity interest in GliaCure Inc. and N.D. founded Sarissa Biomedical.

Correspondence should be addressed to Philip G. Haydon, Department of Neuroscience, Tufts University Medical School, 136 Harrison Avenue, Boston, Massachusetts, 02111. E-mail: Philip.Haydon@tufts.edu.

DOI:10.1523/JNEUROSCI.5689-11.2012

Copyright $\odot 2012$ the authors $\quad 0270-6474 / 12 / 324417-09 \$ 15.00 / 0$
}

plasmic domain of synaptobrevin II (dnSNARE) (Halassa et al., 2009). Because astrocytes are known to release a variety of substances including ATP (Guthrie et al., 1999), which is rapidly hydrolyzed to adenosine in the extracellular space (Dunwiddie et al., 1997), these findings suggest that astrocytes are essential for the wakefulness-dependent increase of extracellular adenosine.

While the effect of adenosine on sleep drive is known to involve direct inhibition of wake-active cholinergic neurons projecting from the basal forebrain (Thakkar et al., 2003), recent findings have suggested that increased adorA1R activation following sleep deprivation may directly impact plasticity in the hippocampus (Florian et al., 2011). Consistent with this idea, we have demonstrated that astrocyte-derived adenosine regulates the surface expression of NMDA receptors (Deng et al., 2011), a key player in many types of synaptic plasticity (Malenka and Bear, 2004). These findings suggest that astrocyte-derived adenosine is involved in the effects of sleep deprivation on memory consolidation; however, it is not known whether the level of astrocytederived adenosine is increased by wakefulness or whether such elevations occur in brain regions relevant to memory formation (Halassa and Haydon, 2010; Porkka-Heiskanen and Kalinchuk, 2011). We therefore determined whether wakefulness induces an increase in extracellular adenosine within the hippocampus and whether wakefulness-dependent elevation of adenosine requires SNARE-mediated vesicular release from astrocytes. Using a combination of electrophysiological, electrochemical, and genetic approaches, we show that wakefulness regulates extracellular adenosine levels through an astrocytic SNARE-dependent mechanism, and that this glial-derived adenosine modulates synaptic transmission and neural circuit function. 


\section{Materials and Methods}

Animals. All procedures were in strict accordance with the National Institutes of Health Guide for the Care and Use of Laboratory Animals and were approved by the Tufts University Institutional Animal Care and Use Committee. Animals were maintained on a $12 \mathrm{~h}$ light/dark cycle before use. The details of the GFAP.tTA and tetO.dnSNARE lines have been previously described (Pascual et al., 2005). Bigenic offspring of crosses (dnSNARE), and their wild-type (WT) littermates, were used in this study. All mice were maintained on $40 \mathrm{mg} / \mathrm{kg}$ Dox containing food (BioServ) until weaning, after which they were switched to a regular diet to allow transgene expression. A total of $156 \mathrm{C} 57 \mathrm{BL} / 6 \mathrm{~J}$ mice (108 WT and 48 dnSNARE) of either sex were used in this study.

Acute hippocampal slice preparation. Horizontal slices were obtained from 8- to 12-week-old mice following brief anesthesia using isoflurane. The brain was rapidly extracted in cold cutting solution that contained the following (in mM): $124 \mathrm{NaCl}, 26 \mathrm{NaHCO}_{3}, 1 \mathrm{NaH}_{2} \mathrm{PO}_{4}, 10$ glucose, 1 sodium pyruvate, 0.6 ascorbate, $2.9 \mathrm{KCl}, 1 \mathrm{CaCl}_{2}$, and $2 \mathrm{MgCl}_{2}$ and bubbled continuously with a $95 \% \mathrm{O}_{2} 5 \% \mathrm{CO}_{2}$ gas mixture. Either $310-\mu \mathrm{m}-$ thick slices (for field recordings) or 400 - $\mu \mathrm{m}$-thick slices (for biosensor recordings) were cut using a vibratome (VT 1200S; Leica). Hippocampal slices were transferred to a bath containing continuously oxygenated cutting solution and incubated at $30^{\circ} \mathrm{C}$ for $1.5 \mathrm{~h}$ before recording. Zeitgeber time (ZT) corresponds to the time of sacrifice.

Extracellular recordings. All field extracellular postsynaptic potential (fEPSP) recordings were made in recording artificial CSF (aCSF) contained the following (in mM): $124 \mathrm{NaCl}, 26 \mathrm{NaHCO}_{3}, 1 \mathrm{NaH}_{2} \mathrm{PO}_{4}, 10$ glucose, 1 sodium pyruvate, $2.9 \mathrm{KCl}, 2 \mathrm{CaCl}_{2}$, and $1 \mathrm{MgCl}_{2}$ and which was continuously superfused at $1.2 \mathrm{ml} / \mathrm{min}$ and maintained at $32.8^{\circ} \mathrm{C}$. Glass recording electrodes filled with aCSF were positioned in area CA1. Signals were amplified and bandpass filtered between $0.1 \mathrm{~Hz}$ and $1 \mathrm{kHz}$ using an 1800 microelectrode amplifier (A-M Systems) and digitized at 5 $\mathrm{kHz}$ via a Digidata 1320 digitizer (Molecular Devices). The CA1-CA2 boundary was severed and paired stimulation pulses were delivered using a $125 \mu \mathrm{m}$ concentric Pt-Ir electrode in the stratum radiatum to orthodromically stimulate axons entering area CA1 $(0.033 \mathrm{~Hz})$. Recordings were acquired with Clampex 9.2 software and analyzed using Clampfit (Molecular Devices).

Estimation of tonic adenosine A1 receptor-mediated inhibition (adenosine tone). The slope of fEPSP was measured online and stimulus intensity/response curves were obtained by supplying stimulating currents of increasing intensity between 50 and $175 \mu \mathrm{A}$ to estimate the maximum slope at saturation. Paired pulse recordings were made throughout the experiment with an interstimulus interval of $25 \mathrm{~ms}$ between pulses within each sweep. Following a $30 \mathrm{~min}$ baseline recording period, inhibitory tone mediated by AdorA1R was estimated by perfusing the slice with the AdorA1R antagonist 8-cyclopentyl-1,3-dimethylxanthine (CPT; 200 $\mathrm{nM}$ ). The increase in the slope of the field potential following CPT application was used to estimate the degree of tonic inhibition (adenosine tone) according to the following equation: Adenosine Tone $=([$ fEPSP. cpt - fEPSP.acsf]/fEPSP.cpt).

In vivo extracellular recordings. Adult mice aged between 6 and 14 weeks were anesthetized by an intraperitoneal injection of urethane (2 $\mathrm{g} / \mathrm{kg}$ ) in two doses over a $1 \mathrm{~h}$ period. Body temperature was measured with a rectal probe and kept at $37^{\circ} \mathrm{C}$ with a heating pad. Depth of anesthesia was assured by continuously monitoring respiration rate, eyelid reflex, vibrissae movements, and testing reactions to tail and toe pinching. Initially, an incision was made, the skull exposed, and a metal plate was adhered with cyanoacrylate glue onto the skull for head fixation. A craniotomy $(1.8 \mathrm{~mm})$ was then drilled in the skull overlaying the somatosensory cortex. The surface of the cortex was kept moist with normal HEPES-buffered artificial CSF that contained the following: (in mM): 125 $\mathrm{NaCl}, 5 \mathrm{KCl}, 5$ glutamate, $10 \mathrm{HEPES}, 3.1 \mathrm{CaCl}_{2}$, and $1.3 \mathrm{MgCl}_{2}$ titrated to $\mathrm{pH} 7.4$ using $1 \mathrm{~m} \mathrm{NaOH}$. The dura was then carefully dissected to expose the cranial surface. Local field potentials (LFPs) were recorded with custom-built electrodes made of two parallel tungsten electrodes which were positioned to record from the superficial layers of the somatosensory cortex. Signals were amplified with an AM-amplifier (AM-Systems), filtered at $0.1 \mathrm{~Hz}$ to $10 \mathrm{kHz}$, and digitized at $50 \mathrm{kHz}$. Recordings for baseline were started at least $10 \mathrm{~min}$ after the electrodes were inserted in the cortex to allow signal stabilization. Recordings were acquired using Clampex 9.2 software (Molecular Devices).

Analysis of in vivo extracellular recordings. For LFP analysis data were sampled at $10 \mathrm{kHz}$ and low-pass filtered at $100 \mathrm{~Hz}$. Power spectra were obtained by averaging a rectangular window over a time period of either 5-10 min (for cumulative effects after $20 \mathrm{~min}$ ) or $27 \mathrm{~s}$ (for timedependent evolution analysis). Power spectra after a given compound was applied were calculated $20 \mathrm{~min}$ after drug application to the surface of the cortex for comparison of overall effects. Power spectra were normalized by the average power at each frequency in the baseline recording period before drug application. Slow oscillation power was calculated by integrating the power spectrum between 0.36 and $1.09 \mathrm{~Hz}$ (slow oscillation range, $0.4-1 \mathrm{~Hz}$ ). To determine whether slow oscillations showed time-dependent evolution in the absence of CPT, we tested whether the baseline slow oscillation power was correlated with time using Spearman's rank correlation test of the normalized baseline. We observed that a subset of recordings in each group showed apparent baseline instability and an upward drift in slow oscillation power (1-2 per group, 6 of 24 total). To avoid potential bias in evaluating the effect of CPT, we did not retain these values in subsequent analysis. Analysis was performed using Clampfit (Molecular Devices) and SigmaPlot software (Systat).

Adenosine biosensor recording. Biosensor electrodes (Sarissa Biomedical) coated with an enzymatic matrix surrounding a platinum electrode (50 $\mu \mathrm{m}$ diameter) were polarized to $+500 \mathrm{mV}$. Electrochemical detection occurred via amperometric measurement of hydrogen peroxide produced by the degradation reaction mediated by the enzymes included in the matrix (Frenguelli et al., 2003; Llaudet et al., 2003). To control for electrical noise and nonspecific electrochemical signal, two sets of biosensor were employed. Adenosine (ADO) biosensors were coated in an enzymatic layer containing nucleoside phosphorylase, xanthine oxidase, and adenosine deaminase while inosine (INO) biosensors lacked adenosine deaminase and were therefore insensitive to adenosine. Before use, all electrodes were hydrated and precalibrated with $10 \mu \mathrm{M}$ adenosine in aCSF that contained the following (in $\mathrm{mm}$ ): $124 \mathrm{NaCl}, 26 \mathrm{NaHCO}_{3}, 1$ $\mathrm{NaH}_{2} \mathrm{PO}_{4}, 10$ glucose, $2.9 \mathrm{KCl}, 2 \mathrm{CaCl}_{2}$, and $1 \mathrm{MgCl}_{2}$. Using these electrode biosensors, adenosine levels were measured in situ in horizontal hippocampal slices. The ADO and INO sensors were inserted sequentially after which the slice was allowed a stabilization period of at least 20 min to avoid aberrant signal produced by acute damage following sensor insertion. Post calibration with $10 \mu \mathrm{M}$ adenosine standard was used to scale adenosine signal. Inosine at $10 \mu \mathrm{M}$ was also applied at the end of experiment to calibrate the relative sensitivity of INO and ADO biosensors to inosine. Estimation of tonic extracellular adenosine was made using INO subtracted ADO signals following the 20 min stabilization period. The value was scaled to the postcalibration standard to estimate concentration. Potentiostat-based recordings were made using the ME200+ Duo-Stat (Sycopel International.) and were digitized via a Digidata 1320 digitizer (Molecular Devices). Acquisition and analysis used Clampex 9.2 software (Molecular Devices). During recording slices were perfused with aCSF at a rate of $1.2 \mathrm{ml} / \mathrm{min}$ with temperature maintained at $32.5-33^{\circ} \mathrm{C}$.

Sleep deprivation. We used the well characterized method of gentle handling to sleep deprive mice. Previous studies have demonstrated that this method effectively reduces the sleep of mice (Halassa et al., 2009). During sleep deprivation, mice were continuously monitored by experimenter over the course of the sleep-deprivation period (4-6h). When mice assumed a sleep posture, the bedding was gently disturbed and redistributed to wake the mouse. If this failed to awaken the mouse, a laboratory glove was used to gently brush the mouse until it awoke. Mice were not habituated to handling before deprivation to reduce stress effects (Longordo et al., 2011).

Analysis of biosensor signal. As the sensitivity of both INO and ADO electrodes are linearly proportional to inosine and the INO biosensor is insensitive to adenosine (Dale et al., 2000; Frenguelli et al., 2003), we corrected the raw signals to account for different sensitivities of the paired electrodes. To do this we adjusted the inosine signal using the ratio of the sensitivity of the ADO and INO electrodes to the inosine standard. The resulting INO response was then subtracted from the ADO signal to 
A
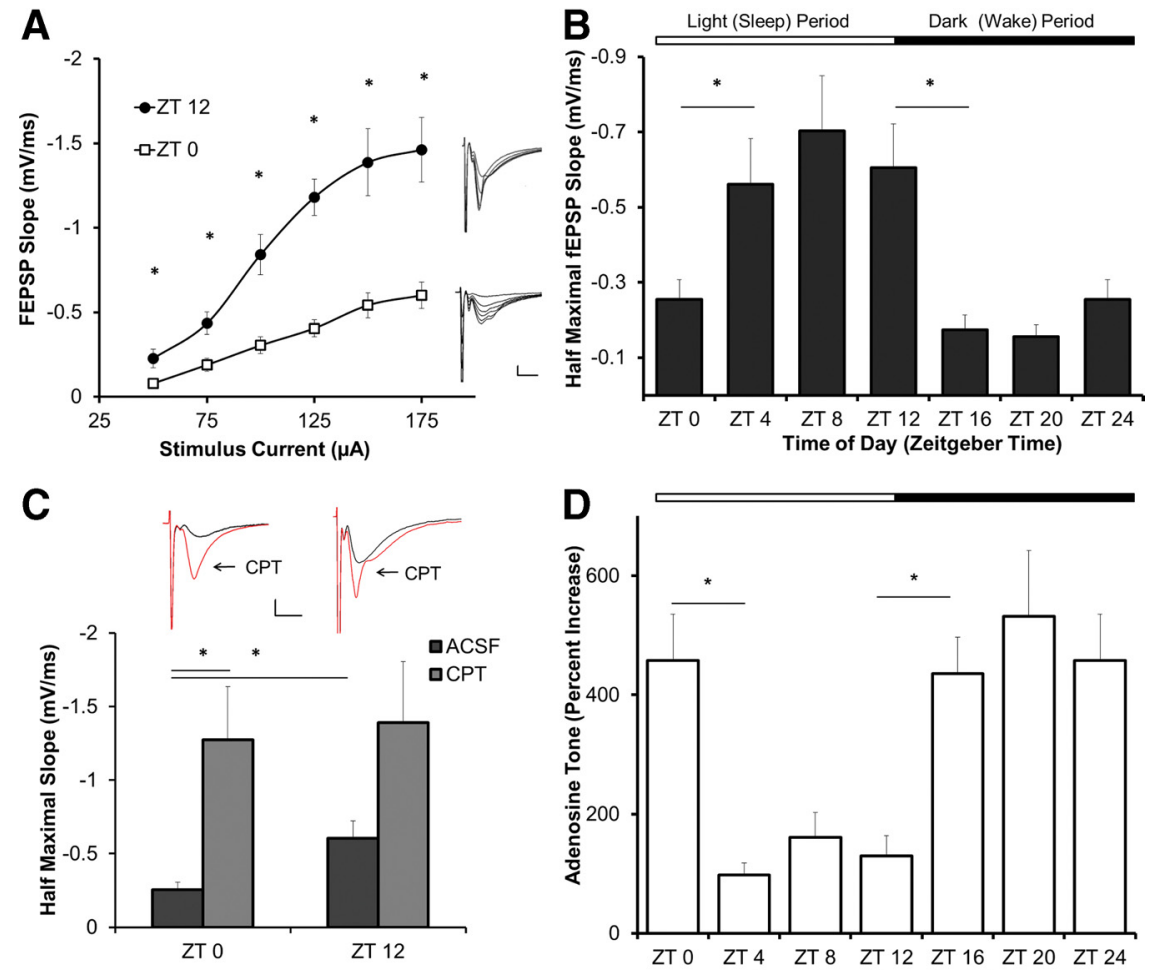

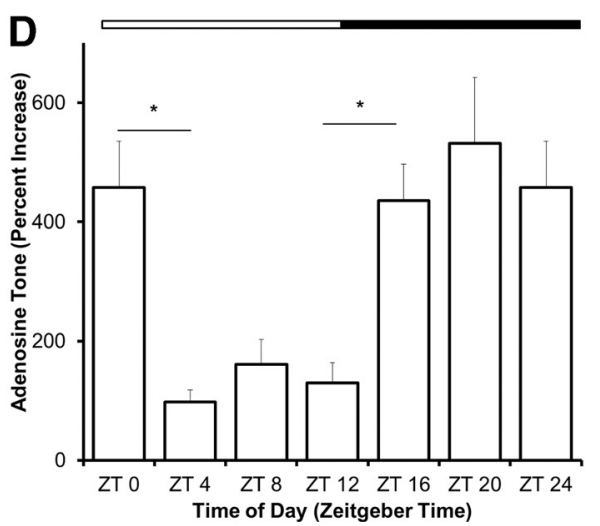

Figure 1. Diurnal changes in adorA1 receptor-mediated inhibition of excitatory synaptic transmission. $\boldsymbol{A}$, fEPSPs slopes were significantly greater in slices taken following the normal sleep period (ZT 12) compared with slices taken following wakefulness (ZT 0) $p<0.005$ (two-way, repeated-measures ANOVA, post hoc SNK $t$ test; ${ }^{*} p<0.05, n=12$ ). Inset, Typical fEPSP traces recorded at increasing stimulus currents. Scale bar, $0.5 \mathrm{mV}, 5 \mathrm{~ms}$. $\boldsymbol{B}$, Slices obtained during the dark phase showed consistently lower half-maximal fEPSP slopes relative to slices taken during the light phase ( $p<0.005$, ANOVA post hoc SNK $t$ test; ${ }^{*} p<0.05, n=$ 6). $C$, The adorA1R antagonist CPT eliminated differences in fEPSP from slices obtained at ZT $=0$ and ZT $=12(p<0.001$, ANOVA; Tukey's test; ${ }^{*} p<0.05, n=6$ ). Inset, Typical half-maximal fEPSP traces before (black) or following (red) application of CPT. Scale bar, $0.5 \mathrm{mV}, 5 \mathrm{~ms}$. D. Tonic adorA1 receptor-mediated inhibition was significantly greater in slices taken in the dark compared with the light phase ( $p<0.001$, Kruskal-Wallis nonparametric ANOVA; Tukey's test; ${ }^{*} p<0.05, n=6$ ).

obtain the current response specific to adenosine. Finally, this signal is calibrated to the adenosine standard to provide the concentration estimate. The capacitative drift in the raw signals was determined based on a first order exponential fit of the signal measured in the slice chamber before placement of the electrodes and was subtracted before scaling. Signal analysis was performed using SigmaPlot (Systat) and MATLAB computing software (MathWorks).

Statistical analysis. Error bars correspond to SEs of the mean (SEM) and $n$ values correspond to the number of animals for all experiments. Statistical analysis was performed in SigmaPlot 11 Software. Group comparisons with multiple groups were initially analyzed with appropriate ANOVA testing followed by Tukey's or Student Neuman-Keuls $t$ (SNK $t$ test) post hoc tests for individual comparisons. Normalized measures were compared using Mann-Whitney $U$ test with Bonferroni's correction or nonparametric ANOVA to account for multiple testing.

\section{Results}

Increased activation of adenosine $\mathrm{A} 1$ receptors following normal wakefulness

To determine the impact of wakefulness-dependent adenosine signaling on hippocampal synaptic transmission, we initially assessed the baseline synaptic properties in hippocampal slices taken from mice at different time points in their normal sleep/ wake cycle. We recorded fEPSPs in slices taken from mice killed at $4 \mathrm{~h}$ intervals during the light/dark cycle. Zeitgeber time 0 ( $\mathrm{ZT}=$ 0 ) corresponds to the onset of the light phase (subjective night time) and ZT 12 to the onset of the dark phase (subjective daytime). Input-output curves elicited in stratum radiatum of area
CA1 revealed that the magnitude of the fEPSP slope was greater for all stimulating currents at the end of the light phase $(\mathrm{ZT}=12)$ compared with the end of the dark phase $(\mathrm{ZT}=0$; Fig. $1 \mathrm{~A})$. Comparison of half-maximal fEPSP slope recordings showed consistently reduced responses during the dark phase which transitioned to a higher response slope after the first $4 \mathrm{~h}$ of the light phase (Fig. $1 B$ ). No difference was observed for half-maximal presynaptic volley amplitude in slices taken at $\mathrm{ZT}=0(0.27 \pm 0.03 \mathrm{mV})$ compared with those taken at $\mathrm{ZT}=12(0.32 \pm 0.06, p=$ 0.46 SNK $t$ test, $n=6$ ) suggesting that changes in axonal conduction do not contribute to the change in fEPSP slope. The paired pulse ratio of fEPSP slope in slices taken at the end of the dark phase $(\mathrm{ZT}=0$; $2.08 \pm 0.15)$ was significantly greater than that obtained from slices harvested at the end of the light phase $(\mathrm{ZT}=12 ; 1.53 \pm$ $0.15, p<0.05$ Mann-Whitney $U$ test) consistent with the idea that the change in fEPSP slope was caused by an increase in presynaptic inhibition.

Because net extracellular adenosine has been shown to rise in the dark phase during wakefulness in the cortex and basal forebrain (Kalinchuk et al., 2011), and because adenosine is known to cause a presynaptic adorA1 receptor-dependent inhibition of synaptic transmission (Cunha et al., 1998), we determined whether the level of adorA1 receptor activation in hippocampal slices was changed as a function of the sleep/wake cycle. To isolate the pool of tonic extracellular adenosine acting on hippocampal adorA1 receptors, we used an in situ adenosine tone assay in acutely harvested slices. Inhibitory adenosine tone was estimated by applying CPT (200 nM), an adorAlR specific antagonist, and measuring the increase in slope of fEPSP. Slices were harvested from mice killed at $4 \mathrm{~h}$ intervals across the sleep/wake cycle starting at the onset of sleep $(\mathrm{ZT}=0)$. Consistent with the previously measured input-output curves, the half-maximal fEPSP slope was significantly lower in slices harvested following the normal period of activity $(\mathrm{ZT}=0)$ compared with slices taken following the normal sleep period $(\mathrm{ZT}=12)$; however, this difference was absent following application of CPT (Fig. 1C). Application of $\mathrm{CPT}$ also significantly reduced the paired pulse ratios in both groups of slices (ZT 0: $2.08 \pm 0.15-1.30 \pm 0.15$ in CPT; ZT 12: $1.53 \pm 0.15-1.13 \pm 0.07$ in CPT). There was no significant difference between the paired pulse facilitation following application of CPT ( $p=0.59$, Mann-Whitney $U$ test) between slices obtained at $\mathrm{ZT}=0$ and $\mathrm{ZT}=12$ further suggesting that adorA1 receptor activation is responsible for the increased presynaptic inhibition observed in ZT 0 slices. The magnitude of basal synaptic transmission was inversely related to the level of adenosine tone which showed a strong negative correlation with fEPSP slope (Correlation Coefficient $=-0.6, p<0.0005$ Spearman's rank order correlation). Adenosine tone was consistently higher in slices taken during or directly following the dark phase and was significantly lower in slices taken following the first $4 \mathrm{~h}$ of the 

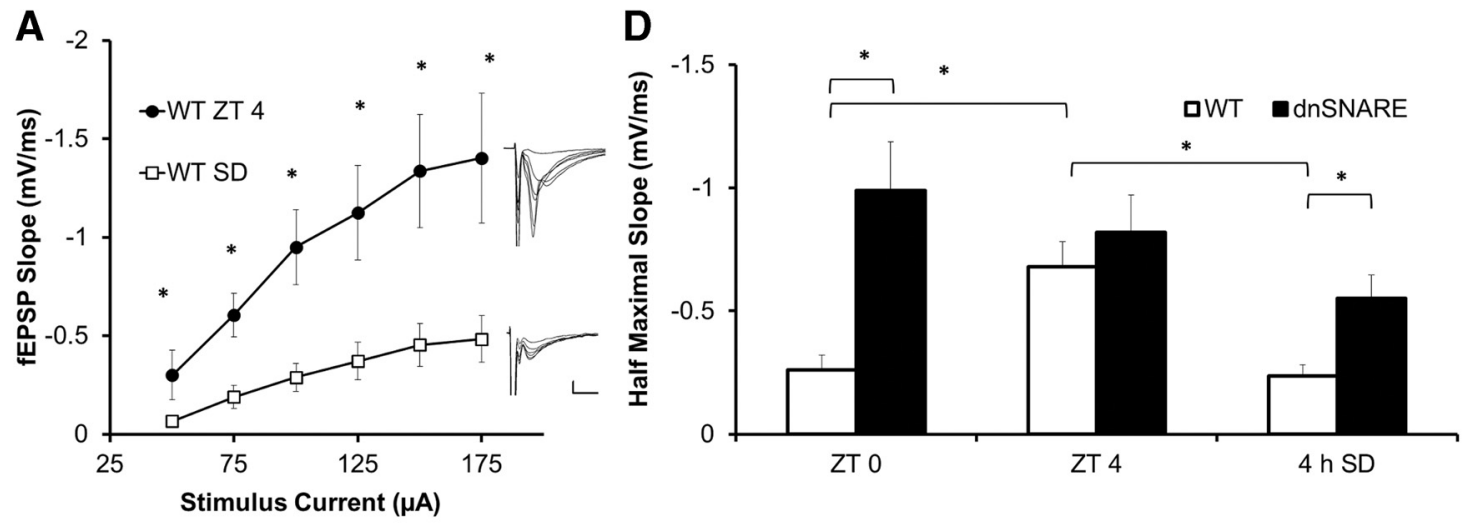

B

E

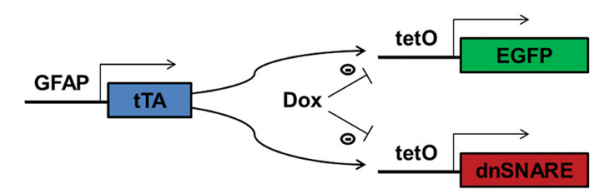

ZT 0
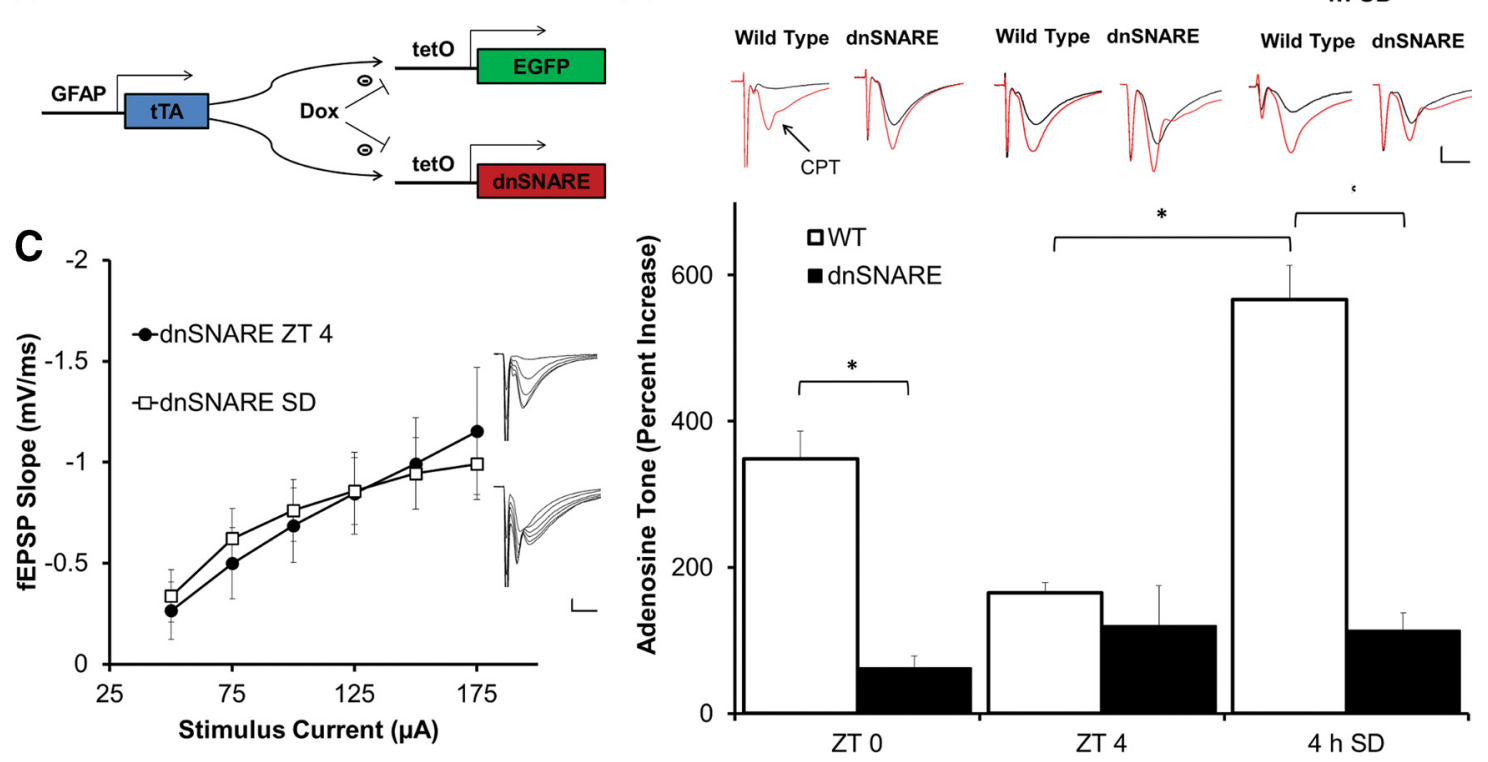

ZT 4

4h SD

Figure 2. Wakefulness-dependent modulation of adorA1 receptor-mediated presynaptic inhibition requires an astrocytic SNARE-sensitive pathway. A, Four hours of sleep deprivation before slice preparation significantly reduced fEPSP slope in WT mice ( $p<0.005$, two-way, repeated-measures ANOVA, posthoc SNK $t$ test; $\left.{ }^{*} p<0.05, n=6\right)$. $\boldsymbol{B}$, Schematic diagram depicting the dnSNARE mouse model which uses conditional dominant-negative suppression of SNARE function under the GFAP promoter to drive the expression of dnSNARE and EGFP (reporter) in astrocytes. $C$, fEPSP slopes in slices from dnSNARE mice were not sensitive to sleep deprivation. Insets, fEPSP traces for increasing intensities of stimulation. Scale bar, $0.5 \mathrm{mV}, 5 \mathrm{~ms}$. D, Half-maximal fEPSP slope was significantly higher in dnSNARE slices taken following normal wakefulness $(Z T=0)$ and following $4 \mathrm{~h}$ of sleep deprivation but not following $4 \mathrm{~h}$ of undisturbed sleep $(p<0.005$, two-way ANOVA, post hoc SNK $t$ test; ${ }^{*} p<0.05, n=6$ ). $E$, Tonic inhibition by adenosine adorA1 receptors was significantly increased by sleep deprivation in slices taken from WT, but not dnSNARE, mice (Mann-Whitney $U$ test; Bonferroni's correction; ${ }^{*} p<0.05 n=6$ ).

sleep period (Fig. 1D). These results suggest that tonic activation of adorA1 receptors is increased during the dark phase (subjective daytime) compared with the light phase (subjective nighttime).

\section{Gliotransmission is necessary for wakefulness-dependent increase in adorA1 receptor activation}

To determine whether the diurnal modulation of fEPSP magnitude and adenosine tone is regulated by wakefulness, we subjected mice to a period of sleep deprivation (induced by gentle handling) between $\mathrm{ZT}=0$ and 4 before cutting hippocampal slices. Input-output curves revealed that sleep deprivation significantly reduced the fEPSP response across stimulation currents in slices taken from WT mice (Fig. 2A).

Previous studies have shown that astrocytes play an important role in the homeostatic response to sleep deprivation (Halassa et al., 2009). The involvement of this cell type is thought to involve the release of ATP, which in the extracellular space is hydrolyzed to adenosine (Dunwiddie et al., 1997), through a mechanism that depends on astrocytic SNARE function (Pascual et al., 2005). We therefore used a line of mice expressing a conditional dominantnegative transgene specifically in astrocytes to disrupt release of transmitters. The transgenic mouse model uses the tetracycline trans-activator from the "tet-off" system (Morozov, 2003) under the GFAP promoter which regulates the expression of "dnSNARE," the cytosolic portion of synaptobrevin II, to disrupt SNARE complex formation specifically in astrocytes (Fig. $2 B$ ). In contrast to results obtained in WT mice (Fig. $2 A$ ), the slope of the fEPSP in slices obtained from dnSNARE mice was insensitive to sleep deprivation (Fig. 2C). The presynaptic volley amplitude at halfmaximal stimulation was similar under both sleep-deprived and undisturbed conditions in both genotypes ( $p=0.38$, two-way ANOVA, $n=6$ ).

Because fEPSP slope in dnSNARE slices is insensitive to prior sleep deprivation, we asked whether the fEPSP is similarly insensitive to diurnal modulation. While the slope of the fEPSP in WT mice significantly increases between $\mathrm{ZT}=0$ and $\mathrm{ZT}=4$, we found that there was no significant difference in fEPSP in dn- 
SNARE slices at these two time points. Expression of dnSNARE significantly increases the fEPSP slope at ZT $=0$ compared with that recorded from WT slices (Fig. 2D). The half-maximal slope was significantly reduced in slices taken from WT but not dnSNARE mice following sleep deprivation, consistent with a wakefulness-dependent effect (Fig. 2D).

Since astrocytic dnSNARE expression is known to lead to reduced adorA1 receptor-dependent presynaptic inhibition of excitatory synapses, we asked whether effects of sleep deprivation were mediated through this glial-dependent signaling pathway. Four hours of sleep deprivation significantly increased the adenosine tone measured in WT slices compared with that measured in WT slices obtained at the same ZT time from undisturbed controls (Fig. 2 E). In contrast to WT, slices taken from dnSNARE mice did not exhibit significant time of day or wakefulnessdependent changes in adenosine tone. For example, adenosine tone was significantly reduced compared with WT slices both after normal wakefulness at ZT 0 and following sleep deprivation (Fig. 2E). Adenosine tone of WT animals was negatively correlated with fEPSP slope (Correlation Coefficient $=-0.69, p<$ 0.005 ) suggesting that synaptic transmission is reduced by increased adorA1 activation. Together, these results suggest that wakefulness increases tonic adenosine-mediated presynaptic inhibition of excitatory synaptic transmission through adorA1 receptors, and that this effect requires an astrocytic dnSNAREsensitive pathway.

\section{Wakefulness increases adorA1 receptor modulation of cortical network activity through an astrocytic SNARE-dependent mechanism}

Measurement of adenosine tone in the hippocampal slice supports the hypothesis that wakefulness increases the level of adenosine-mediated inhibition through a dnSNARE-sensitive mechanism; however, the interpretation of these findings is complicated by the use of an in situ preparation which may affect the levels of adenosine or adorA1R expression and cellular metabolism (zur Nedden et al., 2011). To overcome this potential issue, we next used an in vivo recording method to assess the level of modulation of spontaneous brain activity by adenosine following differing periods of prior wakefulness. We performed LFP recordings in the intact somatosensory cortex of dnSNARE and WT control mice and compared the effect of acute adorA1 receptor inhibition by topical application of $1 \mu \mathrm{M} \mathrm{CPT}$ to the cortical surface.

Initially, we measured the effect of CPT under baseline conditions in mice recorded $6 \mathrm{~h}$ into their normal sleep period (ZT 6). Consistent with previous reports, under urethane anesthesia activity was characterized by synchronized bursts of activity during cortical "up" states interspersed in longer silent episodes or “down" states (Fig. 3A-D, insets). To obtain a nonbiased estimate to quantitatively assess the level of adorA1 receptor-mediated modulation, we performed FFT analysis of the raw signal and compared the resulting power spectrum under baseline conditions with the resulting spectra following application of CPT. The average period of peak synchronized activity was $1-2 \mathrm{~s}$ giving a peak power in the slow oscillation range of $\sim 0.4-1 \mathrm{~Hz}$.

In agreement with measurements in situ (Fig. 2E), tonic adorA1R activation in vivo was low in undisturbed mice during the sleep period $(\mathrm{ZT}=6)$. Addition of CPT to the cortex of undisturbed WT mice did not significantly change slow oscillation power (Fig. 3A). However, prior sleep deprivation potentiated the level of tonic adorA1 receptor activation since application of CPT in sleep-deprived mice enhanced the power of slow oscillations (Fig. 3B). Application of CPT had no effect on slow oscillation power in dnSNARE mice under either undisturbed conditions (Fig. 3C) or following sleep deprivation (Fig. $3 D$ ). Following sleep deprivation, the effect of CPT on slow oscillations was significantly attenuated in dnSNARE mice compared with WT controls (Fig. $3 E$ ). These results demonstrate that extended wakefulness during sleep deprivation increases the modulation of cortical network activity by adorA 1 receptors, and that this increase requires normal SNARE complex formation within astrocytes. Importantly, these in vivo results corroborate our in situ observations by demonstrating that an astrocytic dnSNAREsensitive mechanism is required for forced wakefulness to increase the activation of adorA1 receptors.

\section{Inhibition of SNARE-mediated gliotransmission attenuates wakefulness-dependent increase in basal extracellular adenosine in the hippocampus}

To directly test the hypothesis that astrocytes are required for wakefulness-dependent regulation of extracellular adenosine, we used enzymatic ADO and INO biosensors (Frenguelli et al., 2003; Llaudet et al., 2003). These microelectrode-based biosensors employ sequential enzymatic conversions to produce an electrochemical signal proportional to the concentration of adenosine (Fig. 4A, ADO biosensors) or its major metabolite in the extracellular space, inosine (Fig. $4 A$, INO biosensors). Because ADO biosensors are also sensitive to metabolites of adenosine, we used INO biosensors to isolate the adenosine-specific signal. To compensate for possible differences in the relative sensitivities, we calibrated both sets of biosensors following each experiment with $10 \mu \mathrm{M}$ adenosine and inosine standards and adjusted the differential signal based on the current response of each electrode (Fig. $4 B$ ). Application of increasing concentrations of adenosine demonstrated that the ADO biosensor responded linearly between 0.33 and $10 \mu \mathrm{M}$ adenosine (Fig. $4 C ; R>0.99$ ).

We sequentially positioned the ADO and INO biosensors in contact with the surface of the tissue until a slight deformation of its surface was observed, indicating that the electrode was embedded (Fig. 4D). Comparison of the resulting signal to adenosine and inosine standards allowed us to obtain a quantitative measure of the equilibrium concentration of extracellular adenosine in slices taken from mice under different conditions of prior wakefulness (Fig. $4 E$ ). In slices harvested following normal wakefulness at ZT 0 , the measured concentration of adenosine was significantly higher in WT relative to dnSNARE slices (Fig. $4 E, F)$. To determine whether this increase was an effect of prior wakefulness, we sleep-deprived WT and dnSNARE mice for $6 \mathrm{~h}$ before they were killed and compared slices taken from these animals with others harvested from undisturbed controls. Sleep deprivation significantly increased the level of extracellular adenosine in slices from WT mice; however, this effect was attenuated in slices from dnSNARE mice (Fig. $4 F$ ). Following sleep deprivation, the concentration of adenosine measured in WT slices was comparable to the value measured at ZT 0 and was significantly increased relative to slices taken from sleep-deprived dnSNARE mice (Fig. $4 F$ ). We also observed a trend toward increased levels of adenosine metabolites in sleep-deprived dnSNARE mice $(3.29 \pm 0.74 \mu \mathrm{M})$ compared with WT $(1.51 \pm 0.56 \mu \mathrm{M})$; however, this effect did not reach significance ( $p=0.17, n=6$, SNK $t$ test). These findings support the hypothesis that wakefulness increases the level of extracellular adenosine within the hippocampus through an astrocytic SNARE-dependent mechanism. 


\section{Discussion}

The results of this study provide the first direct measurements of adenosine in the hippocampus during astrocyte specific manipulations. We demonstrate that wakefulness modulates excitatory synaptic transmission by affecting extracellular adenosine derived from an astrocytic source. These results are potentially surprising given that prior microdialysis studies have not measured an effect of sleep deprivation on extracellular adenosine in hippocampus (Zeitzer et al., 2006). It is important to note, however, that microdialysis only captures a proportion of extracellular adenosine. This, coupled with the inherent temporal averaging of in vivo microdialysis, may limit the capacity of this technique to detect wakefulness-dependent changes in adenosine (Strecker et al., 2006; Kalinchuk et al., 2011).

In this study we employ a variety of methods to estimate the level of extracellular adenosine in the CNS, several of which use the acute hippocampal slice preparation. While our findings indicate that this preparation provides a consistent estimate of the level of extracellular adenosine based on both receptor activation and direct concentration measurements, a key caveat to this approach is the use of acute isolated tissue. Although previous studies have demonstrated that incubation of the slice before recording can improve its metabolic state and restore energetic markers, including ATP, to levels comparable to those observed in the intact organism (zur Nedden et al., 2011), it is clear that preparation of the hippocampal slice damages tissue and can affect transmitter release. To address concerns with this preparation, we also performed an in vivo experiment showing that wakefulness-dependent modulation of cortical slow oscillations requires an astrocytic source of adenosine. This finding demonstrates that activation of adorA1 receptors is increased by wakefulness within the cortex through an astrocytedependent mechanism. Although this increase occurs in a different brain region, the pattern is consistent with the wakefulness-dependent elevation measured in situ in the hippocampal slice. Together, these observations strongly suggest that wakefulness biases the equilibrium between release and reuptake of adenosine and that this change is maintained in the hippocampal slice.

Although we demonstrate the importance of a glial source of adenosine for wakefulness-dependent control of extracellular adenosine, our results do not allow us to conclude the mechanism of these daily changes in adenosine. There are many sites that have the potential to regulate extracellular adenosine including wakefulness-dependent changes in release, uptake, or metab$0.05 n=4-5)$.
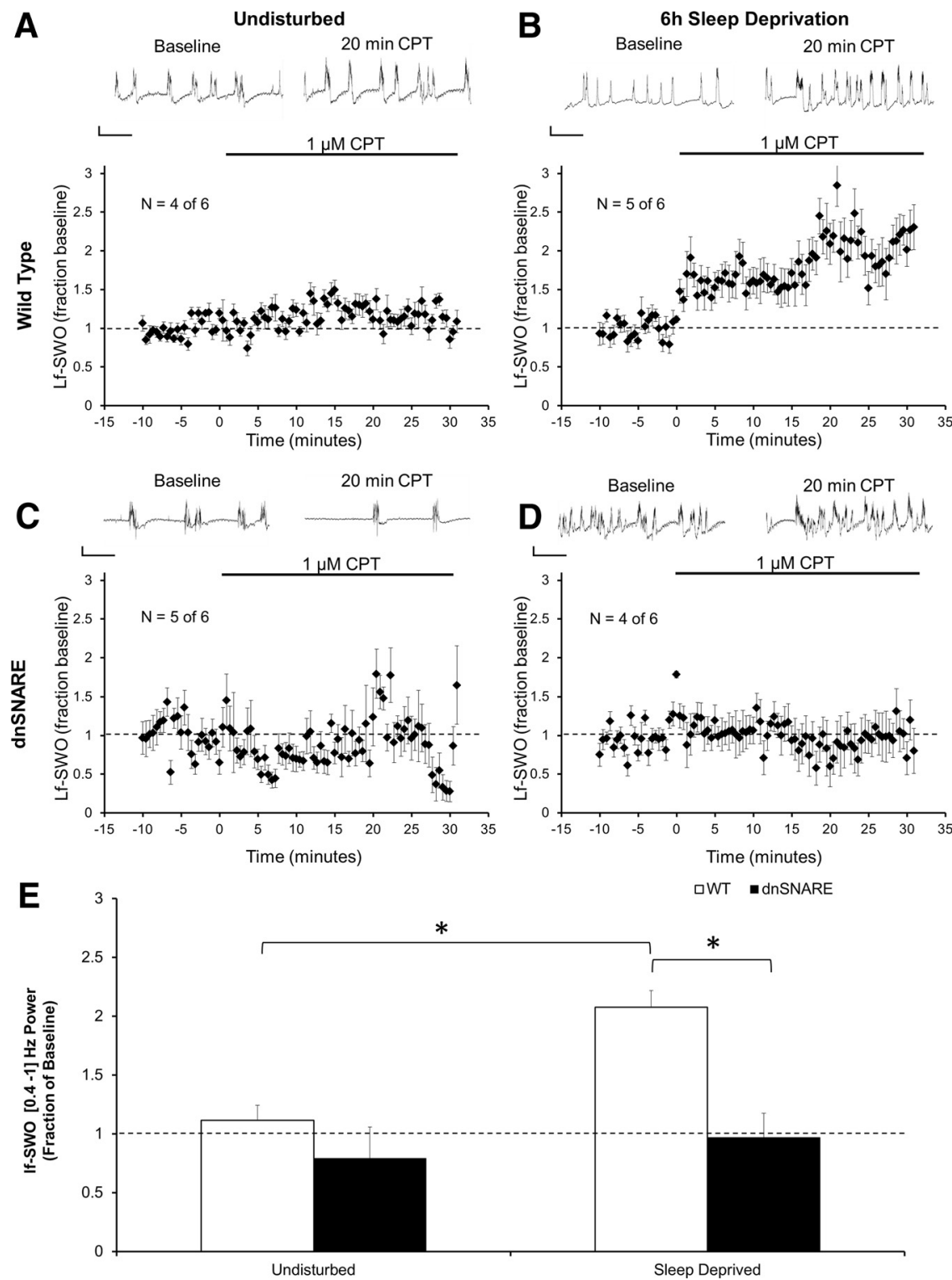

aWT $\because$ dnSNARE

Figure 3. The effects of the adorA1 receptor antagonist CPT on cortical slow oscillations are potentiated by sleep loss through an astrocytic SNARE sensitive pathway. (A-D) LFP recordings were made in the somatosensory cortex of either WT or dnSNARE mice. Application of CPT produced an increase in power of slow oscillations $(0.4-1 \mathrm{~Hz})$ in WT but not dnSNARE mice, and this increase was potentiated by sleep deprivation (FFT of $27 \mathrm{~s}$ spectral segments, normalized to baseline). Inset, Traces show typical LFP recordings during baseline or following (PT ( 30 s recording at time $=22 \mathrm{~min}$ ) Scale bar, $2.5 \mathrm{mV}, 5 \mathrm{~s}$. E, The average increase in slow oscillations following 20 min of CPT was significantly potentiated by sleep deprivation; however, the effect of CPT following sleep deprivation was significantly attenuated in dnSNARE mice (FFT average of 10 min block; Mann-Whitney U test; Bonferroni's correction; ${ }^{*} p<$

olism of adenosine. The locus of this regulation therefore requires further investigation.

The level of tonic adenosine that we have measured is higher than previous estimates of average adenosine concentration in the intact rat hippocampus (Carswell et al., 1997) as well as estimates (Dunwiddie and Diao, 1994) and direct measurements (Frenguelli et al., 2007; zur Nedden et al., 2011) made in rat hippocampal slice. Extrapolations of extracellular adenosine concentration in the hippocampal slice based on pharmacological blockade initially estimated a range of 140 to $200 \mathrm{~nm}$ extracellular adenosine acting on synaptic adorA1 receptors in this preparation. Subsequent direct measurements using inserted biosensors in the rat hippocampus also measured tonic adeno- 
A
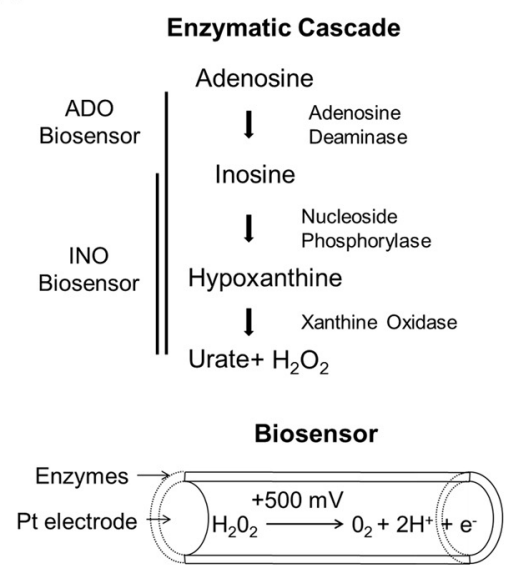

B$$
\text { หุด้ }
$$$$
\text { Adenosine }
$$

Inosine $(10 \mu \mathrm{M})$

$(10 \mu \mathrm{M})$
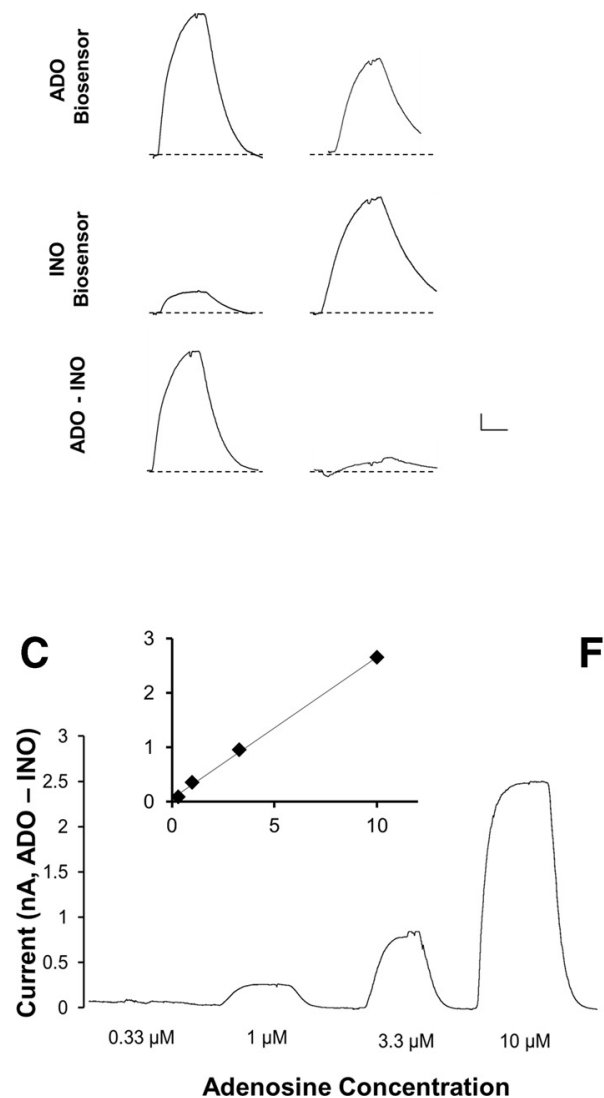

$\mathbf{F}$

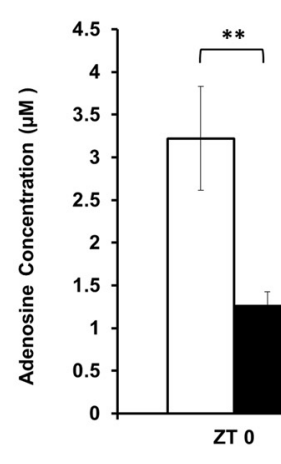

$\mathbf{E}$
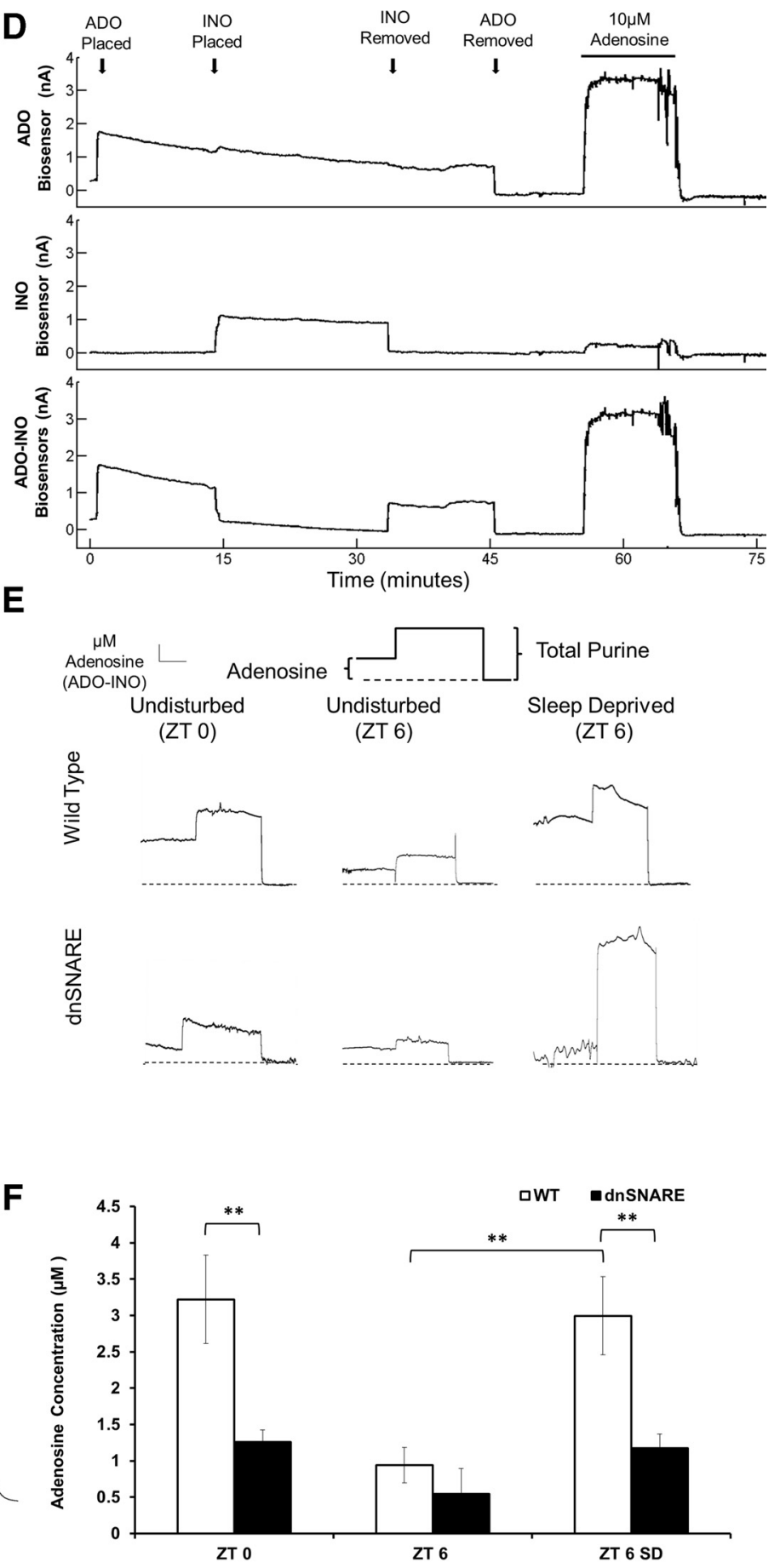

Figure 4. Wakefulness increases the level of hippocampal extracellular adenosine in situ through an astrocyte-dependent mechanism. $\boldsymbol{A}$, Enzymatic conversion of adenosine on the surface of biosensors produces peroxide species which are detected electrochemically. $\boldsymbol{B}$, Traces showing relative sensitivity of adenosine (AD0) and inosine (IN0) electrodes to $10 \mu \mathrm{M}$ ADO (left) and INO (right) standards. Scaled subtraction of the INO signal was used to isolate the adenosine component from other intermediated products in the enzymatic cascade. $\boldsymbol{C}$, Calibration curve of the adenosine biosensor with inosine correction showing linearity over the $0.3-10 \mu \mathrm{m}$ range. $\boldsymbol{D}$, Representative trace of adenosine measurement: AD0 biosensors are lowered into area CA1 followed by IN0 biosensors. Following a 20 min stabilization period, INO biosensors and then ADO biosensors were removed. $\boldsymbol{E}$, Typical sample AD0-INO traces in WT and dnSNARE slices taken following differing conditions of wakefulness. Inset, Diagram shows the segments corresponding to the adenosine signal measured with and without IN0 control electrode insertion into hippocampal area CA1. Samples were scaled relative to postcalibration. Scale-bar, concentration scaled for dual electrode measurement of adenosine, $\mu \mathrm{m} ; 5$ min. $\boldsymbol{F}$, Adenosine was significantly increased in WT but not dnSNARE slices taken following sleep deprivation ( $p<0.005$, two-way ANOVA; Tukey's test; ** $p<0.01, n=6$ ).

sine in this range (Frenguelli et al., 2007) and showed a comparable reduction following inhibition of ectonucleotidase activity suggesting that this adenosine could be derived from extracellular ATP. Interestingly, the concentrations we have measured using similar methods in the mouse preparation are elevated compared with the corresponding values obtained in the rat hippocampus. The relatively high adenosine measured in the mouse may represent a difference in signaling by extracellular adenosine between these rodent species. It is also likely that part of the difference between our measurements and prior estimates is due to a time- 
dependent change in biosensor sensitivity which is not accounted for in the calibration. In this study we employed paired biosensors to differentially measure adenosine while estimating inosine and downstream purine derivatives to which the INO biosensor responds. We observed a consistent change in relative sensitivity of the ADO and INO biosensors over the recording period and, based on the relative change, we estimate that our measurements are elevated by $\sim 60 \%$. Because the procedure is identical under all conditions, however, this differential sensitivity is unlikely to account for the effects of wakefulness or the disruption of wakefulness-dependent changes by dnSNARE transgene expression in astrocytes.

One intriguing aspect of our findings is the observation that adenosine tone appears to rise rapidly within the first $4 \mathrm{~h}$ of wakefulness and to remain relatively stable over the entire dark period. In addition, sleep deprivation maintains, but does not increase, the level of extracellular adenosine measured following spontaneous wakefulness. These observations support a model in which tonic extracellular adenosine does not gradually accumulate during wakefulness in the hippocampus but instead maintains a bistable equilibrium concentration with increased levels during wakefulness that rapidly decline during NREM sleep. Along with the recent demonstration that astrocyte-derived adenosine can increase the surface expression of NMDA receptors (Deng et al., 2011), our results suggest the possibility that elevated adenosine during wakefulness contributes to the enhanced NMDA receptor surface expression observed during wakefulness (Vyazovskiy et al., 2008) thereby supporting experience-dependent plasticity.

Previous studies have shown that the astrocytic expression of the dnSNARE transgene attenuates the negative effects of sleep deprivation on memory consolidation (Halassa et al., 2009), and that the disruption of late-phase long-term potentiation (L-LTP) is prevented in these mice (Florian et al., 2011). The impact of disrupting gliotransmission can be mimicked by application of adorA 1 receptor antagonists, suggesting that these effects are mediated by changes in extracellular adenosine. Having now demonstrated that extracellular adenosine is derived from an astrocytic source, it is possible to speculate about potential mechanisms of the astrocytic modulation of memory consolidation. There are at least two possible pathways to prevent memory consolidation. First, it is known that elevations of cAMP are critical for both L-LTP and long-term memory consolidation (Abel et al., 1997). Since adorA1Rs are $\mathrm{G}_{\mathrm{i}}$-coupled sleep deprivation might prevent memory consolidation by causing an adorA1Rdependent reduction in cAMP. Another possibility concerns the formation of lactate which is an important neuronal energy source and which recent results have implicated as a critical component of memory consolidation (Suzuki et al., 2011). Adenosine can stimulate glycogen synthesis (Daré et al., 2007), which is negatively coupled to lactate production (Brown and Ransom, 2007), leading to reduced lactate availability over time. Interestingly, glycogen synthesis is known to increase following extended sleep deprivation (Franken et al., 2006) suggesting that lactate levels during postdeprivation sleep might be reduced through an adenosine-dependent mechanism. Under conditions of reduced lactate, which is shuttled from an astrocytic source to neurons, memory consolidation and in vivo LTP are impaired (Suzuki et al., 2011). Exogenous application of lactate restores both LTP and memory (Suzuki et al., 2011). Future studies will be needed to discriminate between these two intriguing possibilities.

In conclusion, we have demonstrated that wakefulness enhances the level of adorA1 receptor activation in the hippocam- pus and that this effect is due to a change in the level of extracellular adenosine derived from an astrocytic source. Because adorA1 receptor activation by astrocyte-derived adenosine has been shown to affect both presynaptic release (Latini and Pedata, 2001) and postsynaptic NMDA receptor surface expression (Deng et al., 2011), investigation of the downstream effects of this pathway may reveal important insights into the role of sleep and sleep disruption in regulating hippocampal-dependent memory.

\section{References}

Abel T, Nguyen PV, Barad M, Deuel TA, Kandel ER, Bourtchouladze R (1997) Genetic demonstration of a role for PKA in the late phase of LTP and in hippocampus-based long-term memory. Cell 88:615-626.

Basheer R, Strecker RE, Thakkar MM, McCarley RW (2004) Adenosine and sleep-wake regulation. Prog Neurobiol 73:379-396.

Bjorness TE, Kelly CL, Gao T, Poffenberger V, Greene RW (2009) Control and function of the homeostatic sleep response by adenosine A1 receptors. J Neurosci 29:1267-1276.

Brown AM, Ransom BR (2007) Astrocyte glycogen and brain energy metabolism. Glia 1271:1263-1271.

Carswell HV, Graham DI, Stone TW (1997) Kainate-evoked release of adenosine from the hippocampus of the anaesthetised rat: possible involvement of free radicals. J Neurochem 68:240-247.

Cunha RA, Sebastião AM, Ribeiro JA (1998) Inhibition by ATP of hippocampal synaptic transmission requires localized extracellular catabolism by ecto-nucleotidases into adenosine and channeling to adenosine A1 receptors. J Neurosci 18:1987-1995.

Dale N, Pearson T, Frenguelli BG (2000) Direct measurement of adenosine release during hypoxia in the CAl region of the rat hippocampal slice. J Physiol 526:143-155.

Daré E, Schulte G, Karovic O, Hammarberg C, Fredholm BB (2007) Modulation of glial cell functions by adenosine receptors. Physiol Behav 92:15-20.

Deng Q, Terunuma M, Fellin T, Moss SJ, Haydon PG (2011) Astrocytic activation of $\mathrm{Al}$ receptors regulates the surface expression of NMDA receptors through a Src kinase dependent pathway. Glia 59:1084-1093.

Dunwiddie TV, Diao L (1994) Extracellular adenosine concentrations in hippocampal brain slices and the tonic inhibitory modulation of evoked excitatory responses. J Pharmacol Exp Ther 268:537-545.

Dunwiddie TV, Diao L, Proctor WR (1997) Adenine nucleotides undergo rapid, quantitative conversion to adenosine in the extracellular space in rat hippocampus. J Neurosci 17:7673-7682.

Florian C, Vecsey CG, Halassa MM, Haydon PG, Abel T (2011) Astrocytederived adenosine and A1 receptor activity contribute to sleep lossinduced deficits in hippocampal synaptic plasticity and memory in mice. J Neurosci 31:6956-6962.

Franken P, Gip P, Hagiwara G, Ruby NF, Heller HC (2006) Glycogen content in the cerebral cortex increases with sleep loss in C57BL/6J mice. Neurosci Lett 402:176-179.

Frenguelli BG, Llaudet E, Dale N (2003) High-resolution real-time recording with microelectrode biosensors reveals novel aspects of adenosine release during hypoxia in rat hippocampal slices. J Neurochem 86:1506-1515.

Frenguelli BG, Wigmore G, Llaudet E, Dale N (2007) Temporal and mechanistic dissociation of ATP and adenosine release during ischaemia in the mammalian hippocampus. J Neurochem 101:1400-1413.

Graves LA, Heller EA, Pack AI, Abel T (2003) Sleep deprivation selectively impairs memory consolidation for contextual fear conditioning. Learn Mem 10:168-176.

Guthrie PB, Knappenberger J, Segal M, Bennett MV, Charles AC, Kater SB (1999) ATP released from astrocytes mediates glial calcium waves. J Neurosci 19:520-528.

Halassa MM, Haydon PG (2010) Integrated brain circuits: astrocytic networks modulate neuronal activity and behavior. Annu Rev Physiol 72:335-355

Halassa MM, Florian C, Fellin T, Munoz JR, Lee SY, Abel T, Haydon PG, Frank MG (2009) Astrocytic modulation of sleep homeostasis and cognitive consequences of sleep loss. Neuron 61:213-219.

Kalinchuk AV, McCarley RW, Porkka-Heiskanen T, Basheer R (2011) The time course of adenosine, nitric oxide (NO) and inducible NO synthase 
changes in the brain with sleep loss and their role in the non-rapid eye movement sleep homeostatic cascade. J Neurochem 116:260-272.

Latini S, Pedata F (2001) Adenosine in the central nervous system: release mechanisms and extracellular concentrations. J Neurochem 79:463-484.

Llaudet E, Botting NP, Crayston JA, Dale N (2003) A three-enzyme microelectrode sensor for detecting purine release from central nervous system. Biosens Bioelectron 18:43-52.

Longordo F, Fan J, Steimer T, Kopp C, Lüthi A (2011) Do mice habituate to "gentle handling?" A comparison of resting behavior, corticosterone levels and synaptic function in handled and undisturbed C57BL/6J mice. Sleep 34:679-681.

Malenka RC, Bear MF (2004) LTP and LTD: an embarrassment of riches. Neuron 44:5-21.

Morozov A, Kellendonk C, Simpson E, Tronche F (2003) Using conditional mutagenesis to study the brain. Biol Psychiatry 54:1125-1133.

Pascual O, Casper KB, Kubera C, Zhang J, Revilla-Sanchez R, Sul JY, Takano H, Moss SJ, McCarthy K, Haydon PG (2005) Astrocytic purinergic signaling coordinates synaptic networks. Science 310:113-116.

Porkka-Heiskanen T, Kalinchuk AV (2011) Adenosine, energy metabolism and sleep homeostasis. Sleep Med Rev 15:123-135.

Strecker RE, Basheer R, McKenna JT, McCarley RW (2006) Another chapter in the adenosine story. Sleep 29:426-428.

Suzuki A, Stern SA, Bozdagi O, Huntley GW, Walker RH, Magistretti PJ,
Alberini CM (2011) Astrocyte-neuron lactate transport is required for long-term memory formation. Cell 144:810-823.

Thakkar MM, Winston S, McCarley RW (2003) Al receptor and adenosinergic homeostatic regulation of sleep-wakefulness: effects of antisense to the A1 receptor in the cholinergic basal forebrain. J Neurosci 23:4278-4287.

Van Dort CJ, Baghdoyan HA, Lydic R (2009) Adenosine A(1) and A(2A) receptors in mouse prefrontal cortex modulate acetylcholine release and behavioral arousal. J Neurosci 29:871-881.

Vyazovskiy VV, Cirelli C, Pfister-Genskow M, Faraguna U, Tononi G (2008) Molecular and electrophysiological evidence for net synaptic potentiation in wake and depression in sleep. Nat Neurosci 11:200-208.

Vyazovskiy VV, Olcese U, Hanlon EC, Nir Y, Cirelli C, Tononi G (2011) Local sleep in awake rats. Nature 472:443-447.

Yoo SS, Hu PT, Gujar N, Jolesz FA, Walker MP (2007) A deficit in the ability to form new human memories without sleep. Nat Neurosci 10:385-392.

Zeitzer JM, Morales-Villagran A, Maidment NT, Behnke EJ, Ackerson LC, Lopez-Rodriguez F, Fried I, Engel J Jr, Wilson CL (2006) Extracellular adenosine in the human brain during sleep and sleep deprivation: an in vivo microdialysis study. Sleep 29:455-461.

zur Nedden S, Hawley S, Pentland N, Hardie DG, Doney AS, Frenguelli BG (2011) Intracellular ATP influences synaptic plasticity in area CA1 of rat hippocampus via metabolism to adenosine and activity-dependent activation of adenosine A1 receptors. J Neurosci 31:6221-6234. 